\title{
Computer Numerical Control System of Horizontal Multi-station Cold Heading Machine
}

\author{
Mulan WANG ${ }^{a}$, Hao ZHU ${ }^{b}$, Xiaochun ZHU ${ }^{c}$, Baosheng WANG ${ }^{d}$ \\ ${ }^{a, c}$ Jiangsu Key Laboratory of Advanced Numerical Control Technology, Nanjing Institute of \\ Technology, Nanjing, China 211167 \\ ${ }^{b}$ School of Communication Engineering, Nanjing Institute of Technology, Nanjing, China 211167 \\ ${ }^{\mathrm{d}}$ Research Department of Intelligent Manufacturing Equipment, Nanjing Institute of Technology, \\ Nanjing, China 211167 \\ a wangml@njit.edu.cn, ${ }^{\mathrm{b}}$ zhuhao@njit.edu.cn, ${ }^{\mathrm{c}}$ zhuxc@njit.edu.cn, ${ }^{\mathrm{d}} \mathrm{clxwbs@njit.edu.cn}$
}

\begin{abstract}
Keywords: Cold heading machine, Computer numerical control (CNC) system, Programmable logic controller (PLC), Motor selection

Abstract. In order to overcome the low adjusting efficiency, the bad adaptability and the poor controllability in the conventional mechanical cold heading machine. A kind of novel Computer Numerical Control (CNC) scheme is presented. The overall structure is introduced. The selection methods of main transmission motor and the feeding motor are discussed. The seeking method of the important parameters is detailed described. In order to verify the feasibility of the scheme, the no-load test and normal operating test are performed. The problems in these tests are analyzed and the suggestions of processing are provided. The test after correcting proves that the scheme is accurate and the performance is stable.
\end{abstract}

\section{Introduction}

The horizontal multi-station cold heading machines are popular in modern industrial production because of the advantages of rapid prototyping, simple process and high forging efficiency. With the rapid development of modern science and technology, the market demand for the mechanical products is getting higher. The development trend of mechanical products is to diversification and individualization. The machining curves of cold heading machine are different significantly for the different parts. In the domestic market, most cold heading machines are mechanical control. The main transmission system is consists of motor, flywheel, clutch and crankshaft connecting rod. The rotation of motor is converted to the linear reciprocating motion of slider through the coordination of the flywheel, clutch and crankshaft connecting rod when it working. This motion characteristics is hardly to be precisely controlled and cannot satisfy the requirement of the accurate and complex parts forming. The mechanical components must be exchanged because of the wear of long-term. Besides these, the reactive power of the flywheel and the clutch is high, which accounts for about $50 \%$ of total energy consumption [1]. So the Computer Numerical Control (CNC) system of the cold heading machine can satisfy the requirements of high-precision and flexible in forging processing.

\section{Overall structure of cold heading machine}

The overall structure of cold heading machine and its corresponding control block diagram are shown as Fig.1. The main transmission servo motor and the feeding servo motor are driven by the central controller. The main transmission servo motor connects the crank slider mechanism through the gear reducer. This structure can improve the accuracy of the slider, decrease the transmission error and reduce the energy consumption [2]. At the same time, the structure of gear reducer is much simple and easy to maintenance. Using the gear reducer is help to increase the torque and narrow the motor speed regulation range. The ball screw is driven by feeding motor. Coordinating with the mobile hydraulic clamp and the fixed hydraulic clamp, the feeding process can be accomplished. The precision and the speed of feeding can be increased dramatically by using this structure. The position of slider can be feedback to the controller by the rotary encoder. The controller drive the feeding motor and the hydraulic clamp to work according to the position signal. 


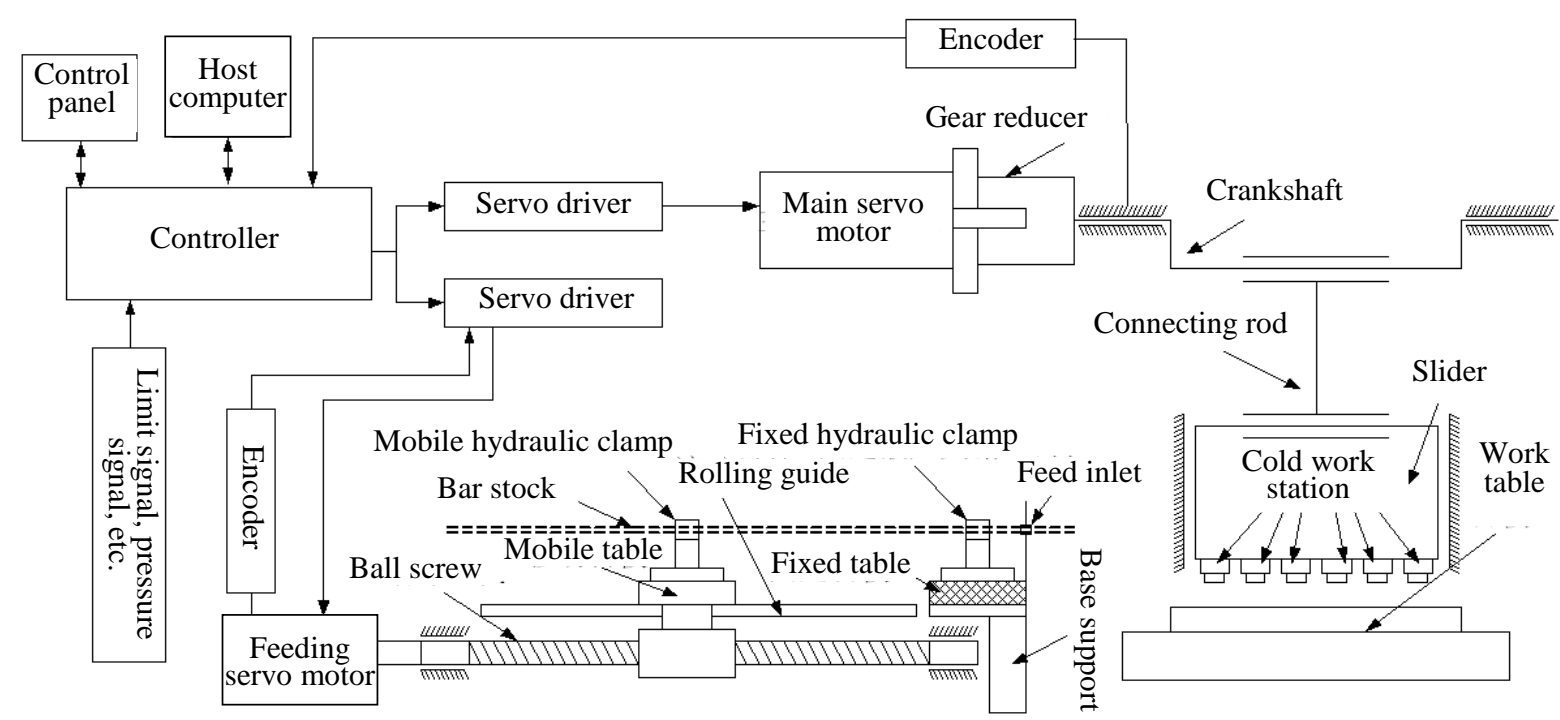

Fig. 1 stucture block of the horizontal cold heading machine

There are two key points in the design as following:

(1) CNC transformation of main transmission system

In this section, the high torque servo motor should be chosen to drive the crankshaft connecting rod directly. The encoder is used to realize the feedback. In this scheme, the slider can reciprocate in the minimum travel to improve the production efficiency. The energy reduction can reach about $50 \%$.

(2) CNC transformation of the feeding system

In this section, the feeding motor must be connected to the ball screw directly and coordinated with the rolling guide to realize the rapid movement of the hydraulic clamp. In this scheme, the feeding length can be controlled accurately. When the feeding motor is controlled by Programmable Logic Control (PLC) and the limit module, the accuracy can reach $0.01 \mathrm{~mm}$.

\section{Control system design}

The function modules of the central control system should including: central controller, digital input/output module, position control module and interface module. The programmable logic controler(PLC) is a kind of industrial control device. The users can expand the capability modules according to their requirments[3]. The advantages of PLC are stable performance, powerful function, wide applicaton, etc. The long time sable operation is necessarily for the cold heading machine. The control accuracy is very high. The quantity of input/output port is large. The lower-end PLC is unable to meet the requirements of such a complex process. PLC of family SIMATIC S7 is the widely used products of SIMENS. S7-300 is used in medium performance system. It allows 32 extension modules. S7-400 is used in high performance system. It can allow more than 300 extension modules. According to the demand of the multi-station cold heading machine, S7-400 is the appropriate control core. The corresponding position module of S7-400 is FM453.

\section{Motor model selection}

Main transmission motor selection. The working characteristics of multi-station cold heading machine is the strong shock-loading and the heavy load. Compare with the conventional mechanical cold heading machine, the larger torque is necessary. Besides this, the stronger anti-interference ability is also needed. The rated power and the rated torque is the most important parameters of the main transmission motor selection [4].

(1) Power calculation

The assumed parameters are as follows: slider stroke $S$, average processing times per minute 40, nominal pressure angle $a$, crank radius $R$, length of connecting rod $L$, reduction gear ratio $i$, cold 
heading force $P_{g}$.

The motor power calculation is to analyze the energy consumption within one work period. The relationship between the slider stroke and deformation force is shown as Fig. 2.

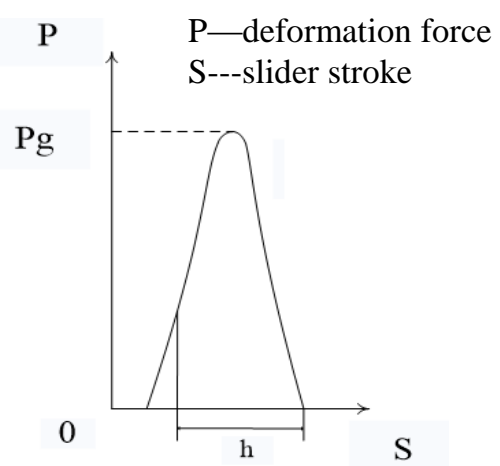

Fig. 2 relationship beteewn the cold heading force and the deformation force

Suppose the energy consumption of the multi-station cold heading machine is $J$. Then:

$$
J=J_{0}+J_{1}+J_{2}+J_{3}+J_{4}
$$

Where $J_{0}$ is the energy of workpiece deformaton, which is effective energy. $J_{1}$ is the energy of driving slider, which is effective energy. $J_{2}$ is the consumed energy of elastic deformation when the slider reach to the limit of the work stroke. $J_{3}$ is the consumed energy of the slider friction. $J_{4}$ is the consumed energy of the slider moving in the empty distance.

1) The energy of workpiece deformation $J_{0}$

The multi-station cold heading machine makes the bar stock deform. Different workpiece needs different energy. When the workpiece is punched, the length after the bar stock deformation is 0.3-0.5 times of the original length. So:

$$
h=0.4 h_{0}
$$

Where $h_{0}$ is the original length, $h$ is the length after deformation.

The curve in the Fig. 2 can be approximated to the triangle. So the energy of workpiece deformation is:

$$
J_{0}=0.7 P_{g} h=0.28 P_{g} h_{0}
$$

2) The energy of driving slider $J_{1}$

The energy of driving slider depending on the cold heading force and the working stroke. Refer to the relative data [4]:

$$
J_{1}=\frac{P_{g} S}{36}
$$

3) The consumed energy of elastic deformation $J_{2}$

In the period from the slider touches the workpiece to leaves the workpiece. The elastic deformation of the machine body and the crank slider generates because of the force, which causes the energy consumption. Suppose all of the elastic deformation energy is consumed:

$$
J_{2}=\frac{1}{2} P_{g} V_{h}
$$

Where $V_{h}$ is the total vertical deformation of cold heading machine, $V_{h}=\frac{P_{g}}{C_{h}} \cdot C_{h}$ is the vertical stifness of cold heading machine, Refer to the relative data, $C_{h}$ of multi-station cold heading machine is normally $400 \mathrm{kN} / \mathrm{mm}$. So:

$$
J_{2}=\frac{1}{8} P_{g}^{2} \times 10^{-8}
$$

4) The consumed energy of elastic deformation $J_{3}$

For the normal cold heading machine, the formula of elastic deformation energy is:

$$
J_{3}=0.5 m_{m} P_{g} a
$$


Where $P_{g}$ is the cold heading force, $m_{m}$ is the equivalent friction force, $a$ is the nominal pressure angle. the formula of $m_{m}$ is

$$
m_{m}=\frac{1}{2} m\left[\left(1+\frac{R}{L}\right) d_{1}+\frac{R}{L} d_{2}+d_{3}\right]
$$

Where the friction coefficient $m=0.045$, the crank diameter $d_{1}=150 \mathrm{~mm}$, the ball-head diameter $d_{2}=140 \mathrm{~mm}$, the crankshaft bearing diameter $d_{3}=120 \mathrm{~mm}$.

$J_{3}$ can be calculated by introducing these parameters into Eq.(8)

$$
J_{3}=\left(3+3.2 \frac{R}{L}\right) P_{g} a \times 10^{-3}
$$

5) The consumed energy of elastic deformation $J_{4}$

Refer to the relative data, the empty distance power of the $60 \mathrm{~T}$ cold heading machine is $1000 \mathrm{~J}$. So

$$
J_{4}=1000
$$

The average power in one work period is:

$$
P=\frac{k J}{t h}
$$

Where $P$ is power, $J$ is consumed energy in one work period, $k=1.5$ is the safety factor, $t$ is the time of one work period.

(2) Torque calculation

According to the force analysis in the Fig. 3, the load torque in the crankshaft $T$ is

$$
T=F_{M N}|O A|
$$

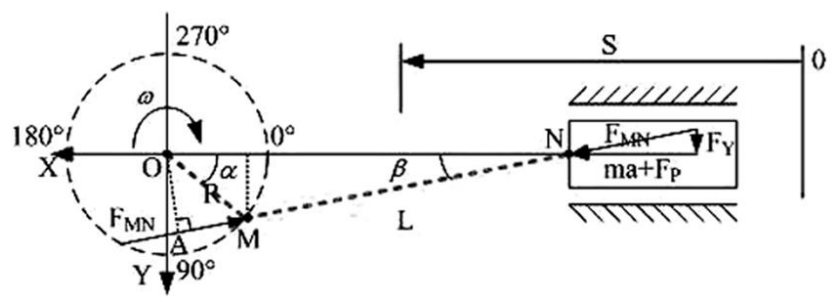

Fig. 3 force analysis of the crank slider

Considering that $b$ is very small

$$
\begin{gathered}
F_{M N}=\frac{P_{g}+m a}{\cos b} \approx P_{g}+m a \\
O A=R \sin (a+b) \approx R \sin \left(a+\frac{l}{2} \sin 2 a\right)
\end{gathered}
$$

Under the condition of good lubrication, the friction torque can be ignored. So the torque which the motor should provide is:

$$
T=\left(\mathrm{P}_{g}+m a\right) R(\sin a+0.5 l \sin 2 a) / i
$$

Where $P_{g}$ is cold force, $i$ is the reduction ratio.

Feeding motor selection. The feeding motor drive the wire rod through the servo motor. The wire rod drive the hydraulic clamp to move. Then the feed process is realized. The load moment in the motor axis is determined by Eq. (16)

$$
T_{m}=\frac{F \times L}{2 p h}+T_{f}+T p
$$

Where $F$ is the force which is needed to move a work table along the axis, $L$ is the moving distance of the work table when the motor rotate one circle, $T_{f}$ is the friction torque between the ball rod axis and the motor axis, $T_{p}$ is the torque between the bar stretching and the motor axis.

The value of $F$ depends on the weight of the work table and the friction coefficient:

$$
F=m(M g+f g)
$$


Where $M$ is the weight of the bar stock. $f$ is the lock force, $m$ is the friction coefficient. Supposing that $M=100 \mathrm{~kg}, f=50 \mathrm{~N}, m=0.05$, then:

$$
\begin{gathered}
F=0.05(100 \times 10+50)=52.5 \mathrm{~N} \\
T_{m}=\frac{52.5 \times 0.4 \times 0.01}{2 p 0.9}+2+5=7.03 \mathrm{~N} \cdot \mathrm{m}
\end{gathered}
$$

\section{Debugging and analysis}

Debugging of the control system. The modules such as PLC, position control module, servo driver, switching power supply, etc. Shuld be connect correctly and reasonably. Because that there may be the large voltage shock when the servo motor starting, the power of servo moter should be seperated from the power of PLC to avoid the instability of the PLC. Besides this, the power line should be seperated from the signal line to prevent the interference. The debugging field is shown as Fig. 4

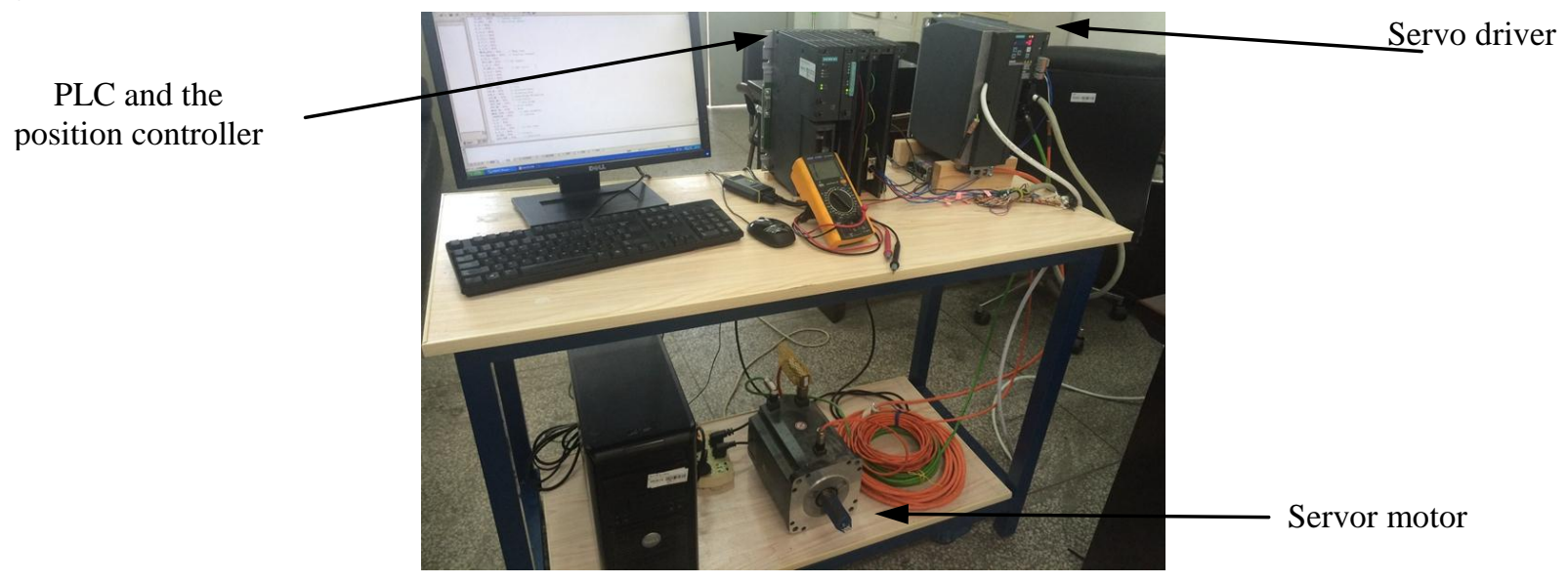

Fig. 4 debugging field of control system

Result Analysis. There are two kinds of the test: the no-load test and normal opertion test. The result analysis is shown as follow:

(1) No-load test

The test includes the realiziation of the feeding process based on the cordination beteewn the mobile hydraulic clamp and the fixed hydraulic clamp, the realization of the punching motion based on the cordination between the main transmission motor and the feeding motor, the position feedback from the encoder to the controller, the working of the elctromagnet and whether the working curve of the slider reach the technological requirements. There are no abnormal problems during the repeated verifications. The no-load test successfuly completed.

(2) Normal operation test.

In this test, there are the phenomenon of crack. After the discussing and analysis, the reason is found. The metals have the characteristics of hardening. The cold heading process belongs to the pressure process. The greater the deformation force of the cold heading machine, the greater the deformation resistance of the parts. There is the maxium deformation degree of the metals. Different metal has different maxium deformation degree. When the deformation exceeds the max value, the cracks appears on the surface of the part. So the reasonable number of the work station should be chosen for the different metal.

The deformation degree is calculated by Eq.(17)

$$
e=\frac{H_{0}-h_{1}}{H_{0}} \times 100 \%
$$

Where $H_{0}$ is original length before cold heading, $h_{l}$ is the length after cold heading. The max deformation degrees of different metals are shown as the table 1 


\begin{tabular}{cc} 
Table 1 max deformation degree of different metal \\
\hline Metal & Max deformation degree $(e)$ \\
\hline $10^{\#} \mathrm{Stl}$ & $80 \%$ \\
$10^{\#} \mathrm{~F} \mathrm{Stl}$ & $87 \%$ \\
$35^{\#} \mathrm{Stl}$ & $73 \%$ \\
$40 \mathrm{Cr}$ Stl & $74 \%$ \\
$30 \mathrm{CrMo} \mathrm{Stl}$ & $55 \%$ \\
$65 \mathrm{Mn} \mathrm{Stl}$ & $50 \%$ \\
$\mathrm{Al}$ & $>95 \%$ \\
$\mathrm{Cu}$ & $>95 \%$ \\
\hline
\end{tabular}

Besides these, the difference of the workpiece shape affects the number of the work station. The general rules are shown as follow:

When the ratio $k$ between the length and diameter of the deformation is extensive, the one-time cold heading will bring the radial bending. The head-form eccentric and local hollow will appear on the molding part. In this situation, the number of the work station can be determined as the following experience:

When $k<2.5$, the times of cold heading is one.

When $2.5<k<4.5$, the times of cold heading is two.

When $4.5<k<6.5$, the times of cold heading is three.

When $6.5<k<8.5$, the times of cold heading is four.

After these correction, the normal operation test is repeated. The phenomenon of crack does not appears. According to these tests, the multi-station cold heading machine can meet the requirements though multiple cold headings with different max deformation degrees.

\section{Conclusions}

The study of driving the cold heading machine directly by the servo motor is still in the initial stage. In this paper, the CNC transformation of the multi-station cold heading machine is detailed researched. The feasibility of the method is verified. With the deepening of the research, some technical problems and weak-points are encountered, such as the function of system monitor. This is discussed in reference [5]. The remote monitoring system for multi-station cold heading machine, which uses the cores of the embedded microprocessor (ARM) and wireless communication module (SIM900) based on the GPRS network communication platform, is provided in this paper.

\section{Acknowledgements}

This work was financially supported by the Industry-university-research Prospective Project of Jiangsu Province (Grant No.: BY2013009-01).

\section{References}

[1] Xu Gaochun. Optimization research and system implimentation for high speed automatic cold heading machine to environment protetion. Shanghai Univesity (2010)

[2] Wang Mulan: Numerical control principle and system. Machinery industry Press (2012)

[3] Gu Yan. Numerical control of multi station horizontal cold heading machine and intelligent monitoring system. Nanjing institute of technology (2015)

[4] Gu Shengu: Motor and drag fundation. Machinery industry Press (2011)

[5] Gu Yan, Wang Mulan, Zhao Xueyao. Remote monitoring system for multi-station cold heading machine based on GPRS. Development and innovation of machinery and electrical products. (2015), p.117-121 